\title{
High-Resolution, Large-Area Fabrication Of Compliant Electrodes Via Laser Ablation For Robust, Stretchable Dielectric Elastomer Actuators And Sensors
}

\author{
O. Araromi; S. Rosset; H. Shea
}

This document is the unedited Author's version of a Submitted Work that was subsequently accepted for publication in ACS Applied Materials and Interfaces, copyright (C) American Chemical Society after peer review. To access the final edited and published work see http://doi.org/10.1021/acsami.5b04975. 


\section{High-resolution, large-area fabrication of compliant}

\section{electrodes via laser ablation for robust, stretchable}

\section{dielectric elastomer actuators and sensors}

Oluwaseun A. Araromi*, Samuel Rosset, Herbert R. Shea*

Microsystems for Space Technologies Laboratory (LMTS), École Polytechnique Fédérale de Lausanne (EPFL), Rue de la Maladière 71B, Neuchatel 2000, Switzerland.

Keywords: Dielectric elastomer sensors, dielectric elastomer actuators, laser ablation, oxygen plasma-bonding, electroactive polymers.

Abstract: A key element in stretchable actuators, sensors and systems based on elastomer materials are compliant electrodes. While there exist many methodologies for fabricating electrodes on dielectric elastomers, very few succeed in achieve high-resolution patterning over large areas. We present a novel approach for the production of mechanically robust, highresolution compliant electrodes for stretchable silicone elastomer actuators and sensors. 2 to 50 $\mu \mathrm{m}$ thick cast poly(dimethylsiloxane)(PDMS)-carbon composite layers are patterned by laser ablation and subsequently bonded to a PDMS membrane by oxygen plasma activation. The technique affords great design flexibility and high-resolution, and readily scales to large area arrays of devices. We validate our methodology by producing arrays of actuators and sensors on 
up to A4-size substrates, reporting on micro-scale dielectric elastomer actuators (DEA) generating area strains of over $25 \%$, and interdigitated capacitive touch sensors with high sensitivity yet insensitivity to substrate stretching. We demonstrate the ability to co-fabricate highly integrated multifunctional transducers using the same process flow, showing the methodology's promise in realizing sophisticated and reliable complex stretchable devices with fine features over large areas.

\section{INTRODUCTION}

In recent years the development of stretchable actuators and sensors based on the deformation of elastomers has received significant interest from researchers creating novel compliant and damage resilient systems, such as bio-inspired sensor skins, ${ }^{1}$ mechanically robust sensors ${ }^{2,3,4}$ high-strain electroactive actuators, ${ }^{5,6}$ or foldable robotics. ${ }^{7,8}$ We present a novel fabrication methodology for the production of large-area, high-resolution and compliant electrodes for deformable and mechanically robust dielectric elastomer actuators (DEAs) and dielectric elastomer sensors (DESs). Our method is based on laser-patterned thin silicone-carbon composite layers bonded to silicone membranes using oxygen plasma activation. This approach combines the high-resolution, high throughput, and large-area processing of laser patterning, with the ability to use nearly any silicone elastomer membrane, while making use of watersoluble sacrificial layers to enable easy handling.

The realization of compliant electrodes is an important aspect in the development of DEAs and DESs. In DEAs, two compliant electrodes sandwich the elastomer membrane (usually a silicone or acrylic elastomer) and the application of a voltage bias (typically several kilovolts) across them causes thickness reduction and in-plane expansion of the membrane due to electrostatic 
forces. ${ }^{5}$ The resulting compressive stress $\sigma$ through the thickness of the elastomer membrane is given by:

$$
\sigma=\varepsilon E^{2}
$$

where $\varepsilon$ is the permittivity of the elastomer membrane and $E$ is the applied electric field. The resulting in-plane strain $e$ can be approximated by (for free boundary conditions):

$$
e=\frac{\varepsilon E^{2}}{Y_{e f f}}
$$

where $Y_{\text {eff }}$ is the effective modulus of elasticity of the electrode-elastomer sandwich expressed by ${ }^{9}$,

$$
Y_{e f f}=\frac{Y_{s} t_{s}+2 Y_{e} t_{e}}{t_{s}+2 t_{e}}
$$

where $Y$ is the elastic modulus, $t$ is the thickness and the subscripts s and e refer to the silicone elastomer and electrodes respectively. From (2) and (3) it is clear that the electrodes must be as thin and compliant as possible i.e. possessing preferably an elastic modulus and thickness much lower than the elastomer, in order to maximize the strain produced in actuation.

In capacitive DESs, strain sensing is performed by detecting changes in the membrane capacitance ${ }^{10}$, and touch sensing is performed by detecting changes in the fringe field between parallel or co-planar electrodes ${ }^{3,11}$. Here the electrode compliance is required to enable the elastic properties of the elastomers to be fully exploited. Additionally electrodes for DEAs and DESs should remain conductive at high strains $(>20 \%)$ and over a large number of cycles $\left(>10^{6}\right)$, and maintaining their mechanical integrity during transduction or in the presence of externally applied mechanical deformations. Numerous electrode technologies for dielectric elastomer 
transducers have been investigated to date, as extensively reviewed in, ${ }^{9}$ including: deposited metallic thin layers, ${ }^{16}$ implanting metallic particles in the elastomer surface, ${ }^{17,18,19}$ carbon nanotube- or metallic nanoparticle-elastomer composites, ${ }^{20,21}$ encapsulated liquid metal micro channels, ${ }^{22,23,24}$ transparent hydrogels and ionogels, ${ }^{25,26}$ and carbon (including graphite) loose powers, greases or composites $,{ }^{27},{ }^{28},{ }^{29}$ which are the most commonly used.

Methods based on metal films are attractive due to their high conductivities $\left(<10 \Omega \mathrm{sq}^{-1}\right)$ but typically over-stiffen the underlying elastomer, though unidirectional actuation has been achieved by micro-structuring the elastomer membrane ${ }^{16}$. Ionic electrode methods (hydrogels and ionogels) are attractive due to their optical transparency but have yet to be demonstrated at high pattern resolution. Carbon based methods have been used extensively by several researchers, for example in the development of actuators capable of large area strains (> $50 \%),{ }^{6,28,29}$ as these materials can be easily manipulated and have a relatively low stiffening impact on the elastomer membrane. Capacitive sensing has also been demonstrated using carbon based electrodes ${ }^{10}$. Carbon powders dispersed in elastomer matrices in particular offer good mechanical robustness and the ability to tune the electrode conductivity.

Silicone elastomers are appealing materials for stretchable transducers, both for use as the elastomer dielectric or as the elastomer matrix in compliant electrodes. They are lightweight, can be processed easily using a variety of methods, ${ }^{12,13}$ and are highly compliant, in some cases capable of being stretched $>1000 \%$ without rupture. Moreover silicone elastomers are available in a wide range of elastic moduli, generally possess low viscous properties leading to fast electromechanical response times, ${ }^{14,15}$ and can, as is the case for poly(dimethylsiloxane)(PDMS), be optically transparent. 
Patterning polymers by laser ablation has been used in the past to fabricate micro-scale features $^{30,31}$ and bonding of PDMS using oxygen plasma has been widely used in the microfluidics domain. ${ }^{32,33}$ In this work we combine these processes together with thin film casting of compliant electrodes to create a novel fabrication method well suited to dielectric elastomer transducer fabrication. By decoupling the electrode patterning and bonding steps our fabrication methodology enables easy scaling to large areas $\left(>100 \mathrm{~cm}^{2}\right)$, for example for the production of device arrays (Figure 1A), whilst maintaining high-resolution features (Figure 1B and Figure 1C). Once the electrode is bonded to the elastomer membrane, the water-soluble substrate is simply dissolved away in hot water (see Video S1 in the supporting information), providing an elegant electrode transfer solution. An additional advantage of our transfer method is the low mechanical stresses imposed on the elastomer membrane compared to other methods such as stamping or manual patterning, hence our process can also be used with ultra-thin elastomer films $(<5 \mu \mathrm{m}$ thickness) without causing rupture. The flexibility in the process facilitates short design iteration loops, allowing transducer designs to be fabricated, tested and optimized quickly. Moreover our technique also facilitates the fabrication of highly integrated, multifunctional dielectric elastomer systems i.e. capable of simultaneous sensing and actuation, on a single elastomer membrane with a single process flow, accelerating the development of sophisticated stretchable devices.

A description of our fabrication method is provided in the experimental section (section 2) along with details of the experiments conducted in this work. Characterization of the fabrication method in terms of adhesion, resistance versus stretch and plasma induced stiffening is provided in section 3.1. We then demonstrate the efficacy of our method in fabricating stretchable micro- 
scale actuators, capacitive touch sensors and multifunctional devices capable of simultaneous actuation and sensing in sections 3.2 and 3.3.

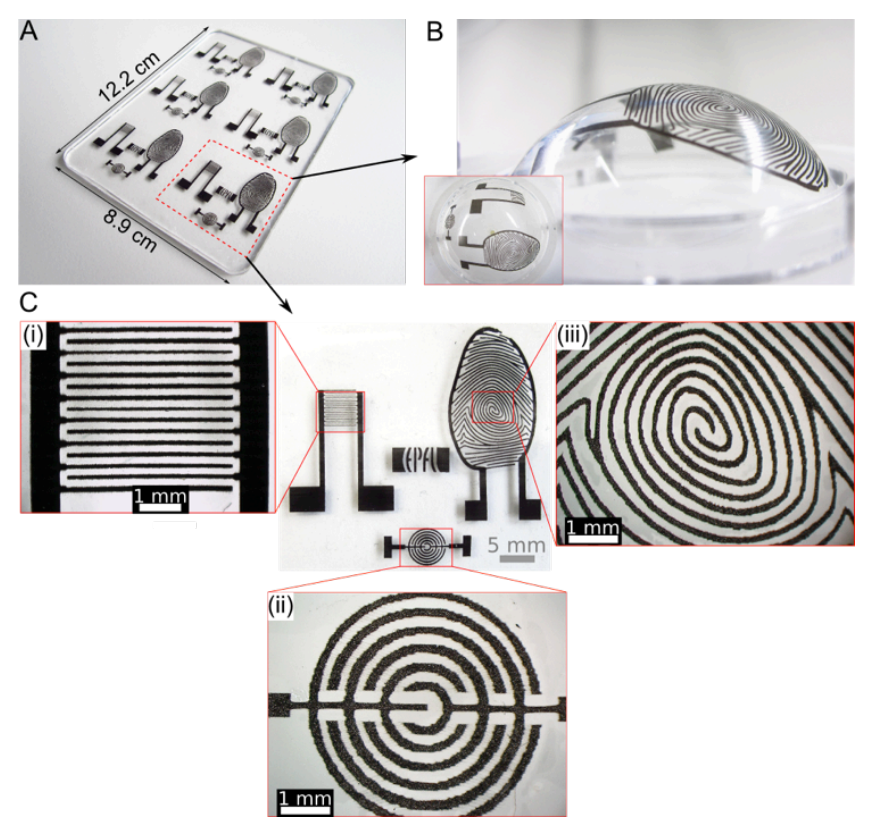

Figure 1. Interdigitated transducer geometries produced by laser-ablation of cast carbon-silicone bonded to an elastomer). A) An array of transducers, bonded to a thick PDMS substrate. B) Side view of transducers bonded to thin PDMS membrane and inflated, demonstrating their high deformability (inset figure, top view). C) Details of the different interdigitated transducer geometries, (i) rectangular, (ii) circular, (iii) fingerprint shape. Resolution for these devices is simply set by the laser-engraver used, not intrinsically by the method.

\section{EXPERIMENTAL SECTION}

Generalized Fabrication Methodology: Our generalized fabrication methodology is outlined in Figure 2. The water-soluble sacrificial substrate is made by casting a polyvinyl alcohol (PVA) aqueous solution onto a poly(ethylene terephthalate) (PET) substrate (Figure 2A) to a width of 
$20 \mathrm{~cm}$ and length of $29 \mathrm{~cm}$. The water in the PVA solution is allowed to evaporate for 12 hours leaving behind a flexible film with a thickness of $40 \mu \mathrm{m}$. The composite electrode was made by ball mixing carbon black (Ketjenblack 300, AkzoNobel) with a soft PDMS (MED4901, NuSil, elastic modulus approximately $0.1 \mathrm{MPa}$ ) at a 1:10 weight ratio. The electrode mixture is cast onto the PVA substrate to the desired thickness (Figure 2B) and subsequently cured in an oven (electrode planar dimensions $15 \mathrm{~cm}$ by $26 \mathrm{~cm}$, typical thickness between $4-8 \mu \mathrm{m}$ ). The cured electrode and PVA substrate are peeled from the PET substrate and patterned by laser ablation using a $\mathrm{CO}_{2}$ laser engraver (Speedy 300, Trotec) (Figure 2C). The laser patterned electrode and a separately prepared silicone membrane are treated in an oxygen plasma using a low-pressure plasma system (Zepto, Diener electronic) (Figure 2D) operating at $13.56 \mathrm{MHz}$, a chamber pressure between $0.2-0.3$ mbar and a power of $20 \mathrm{~W}$. The treated surfaces are then placed in contact with each other to initiate bonding (Figure 2E), a small amount of ethanol is placed between the surfaces prior to placing the them together in order to aid conformal contact. After 30 minutes the PVA substrate of the electrode is removed by dissolving in hot water ((Figure $2 \mathrm{~F})$.

Selection of PDMS in experiments: Sylgard 186 (Dow corning, elastic modulus approximately $1 \mathrm{MPa}$ ) was used as the dielectric in all DEAs experiments as it has been shown to perform well when used for DEAs. ${ }^{7,8,14}$ Sylgard 186 membranes pre-stretched equi-biaxially by 1.33 , with a final thickness of approximately $25 \mu \mathrm{m}$, were used in the plasma induced stiffening and micro-actuator experiments. Membranes with a final thickness of $10 \mu \mathrm{m}$ were used for the multifunction transducer experiments.

MED-4901 was used in the electrode mixture for all experiments, as the substrate in the capacitive sensor experiments and encapsulating material in the multifunctional transducer 
experiments due to its low elastic modulus (approximately $0.1 \mathrm{MPa}$ ) and therefor low stiffening impact.

Resistance vs. strain experiments: For the resistance vs. strain measurements presented in Figure 3, the electrodes were bonded to a thick $(100 \mu \mathrm{m})$ MED-4901 membrane to facilitate handling using an exposure time of $11 \mathrm{~s}$. Resistance data was taken using four-probe measurement to eliminate the effect of contact resistance. A Standa motorized stage (model 8MT175-150) driven at $0.5 \mathrm{~mm} \mathrm{~s}^{-1}$ was used to apply strains. After each measurement run the strain was ramped down to $0 \%$ and the electrodes allowed to recover over a period of at least 15 minutes before repeating the test.

Characterization of plasma induced stiffening: For the characterization of stiffening impact presented in Figure 4, only the region of the PDMS membrane covered by the electrode was exposed to the plasma in order to eliminate the mechanical coupling of the passive region (the region not covered by the electrode). Devices with stamped electrodes made using a TPM101 Teca print pad printing machine (further details on the stamping process are provided in the supporting information) were used for comparison. The elastomer membrane dimensions were $40 \mathrm{~mm}$ wide and $12.8 \mathrm{~mm}$ long (in the actuation direction), electrode dimensions were $30 \mathrm{~mm}$ wide and $4 \mathrm{~mm}$ long. $1 \mathrm{~mm}$ thick PMMA elements laser etched with image recognition markers were bonded to the center of the membranes. The markers were used to track the actuator displacement, which were captured using a USB microscope. Image analysis was performed using a Labview script.

Micro-actuators: For the micro-scale actuators presented in Figure 5, electrode lines of width approximately 100, 200 and $300 \mu \mathrm{m}$ were laser ablated and bonded to the back and front sides of 
the PDMS membrane in a cross pattern using a plasma exposure of $11 \mathrm{~s}$. Input voltages between 0 and $3.0 \mathrm{kV}$ were applied incrementally to the actuators using a high voltage supply (EAP Controller, Auckland Biomimetics Laboratory) and held for $20 \mathrm{~s}$. Pictures of the micro-actuators were taken at each voltage input using an optical microscope and the resulting strain measured using ImageJ software (National Institutes of Health). The area strain was determined using the width strain measured at the center of the top electrode, represented schematically in the inset Figure 5 (assumed to be homogeneous over the active region and equal to the strain in the perpendicular direction.)

Interdigitated capacitive touch sensor: The capacitive sensor presented in section 3.2 was fabricated using an electrode approximately $7 \mu \mathrm{m}$ thick bonded to a $5 \mu \mathrm{m}$ thick MED-4901 membrane using a plasma exposure time of $11 \mathrm{~s}$. The device was subsequently encapsulated using a second, thicker MED-4901 membrane (20 $\mu \mathrm{m})$, using the same plasma parameters, to protect it from contaminants and to facilitate handling. The resulting device is shown in Figure 6A. The sensor was characterized at $0 \%$ and $50 \%$ uniaxial strains in the direction perpendicular to the lines of the interdigitated electrodes (Figure 6B inset). Change in impedance versus separation distance from grounded conductors and high permittivity insulators encapsulated in a PDMS balloon were measured using an Agilent E4980A precision LCR (impedancecapacitance-resistance) meter at $1 \mathrm{kHz}$ measurement frequency (Figure 6B and Figure 6C). Details of the PDMS balloon fabrication and schematic of experimental set-up are provided in the supporting information. A measurement was also taken using a PMMA probe (relative dielectric constant approximately 2) at zero prestretch for comparison.

Multifunction transducer: The multifunctional device presented in section 3.3 was made in two halves, the top half having one of DEA electrodes, the sensor electrodes and an 
encapsulation layer, and the other half having the bottom DEA electrode. The two halves were later bonded together back-to-back using oxygen plasma activation with an exposure time of $11 \mathrm{~s}$ (A schematic cross-section of the device is provided in Figure 7B). This design approach reduced the chance of device failure during actuation due to defects in the dielectric membranes. The electrodes for the device were $7 \mu \mathrm{m}$ thick, the passivation layer for the sensor was a $10 \mu \mathrm{m}$ thick MED-4901 membrane (NuSil) bonded by oxygen plasma using an exposure time of $11 \mathrm{~s}$.

A Labview script was used to monitor the capacitance of the sensor and trigger cyclic actuation of the actuator at $3 \mathrm{~Hz}$ when the capacitance fell below a user specified threshold i.e. when a touch input was detected (Figure 7C). The script was written such that the device operated in latching mode i.e. the device continued actuating periodically until another touch input was detected. The sensor threshold to trigger actuation was set to a reactance of $-325 \mathrm{M} \Omega$, equivalent to $0.48 \mathrm{pF}$ (assuming a series resistance-capacitance circuit model).

\section{RESULTS AND DISCUSSION}

A

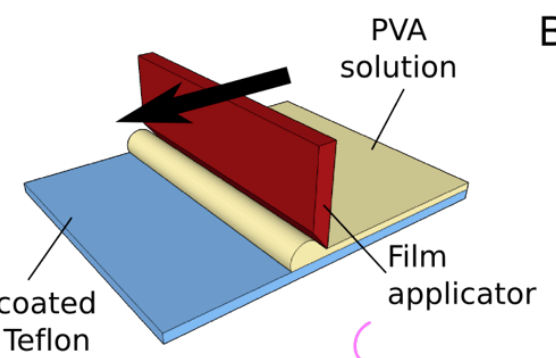

with Teflon

D

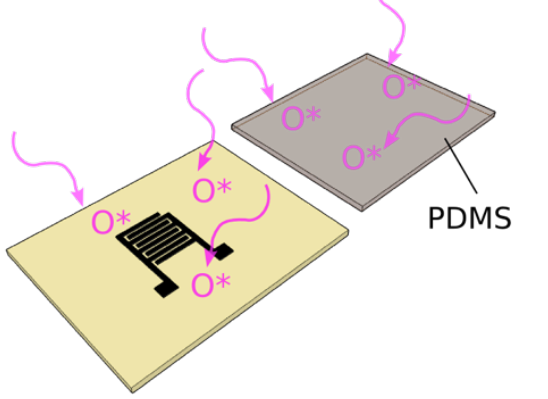

B

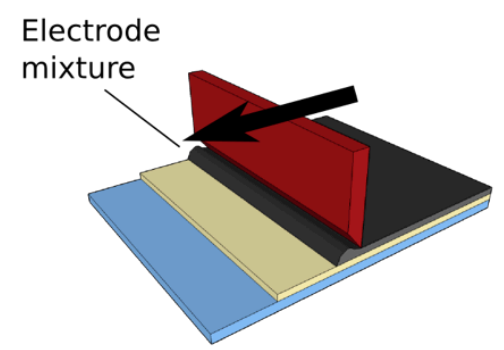

E

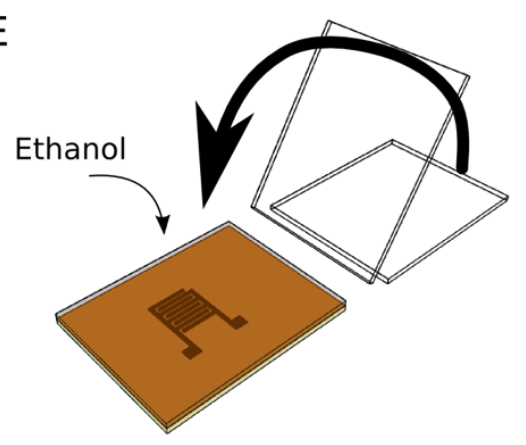

C

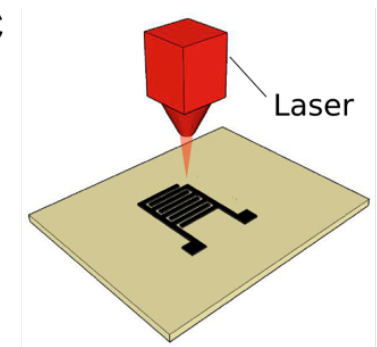

$\mathrm{F}$

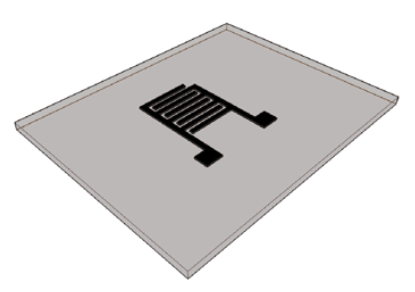


Figure 2. Schematic representation of the general fabrication procedure for compliant electrodes on thin silicone membranes. A) A PVA aqueous solution is cast onto a PET film coated with PTFE (PVA planar dimensions $20 \mathrm{~cm} \times 29 \mathrm{~cm}$ ). B) Casting of uncured electrode (planar dimensions $15 \mathrm{~cm}$ and $26 \mathrm{~cm}$ ). C) The cured electrode (typical thickness between 4-8 $\mu \mathrm{m}$ ) and PVA substrate are released from the PET film and the electrode patterned by laser ablation using a CO2 laser engraver. D) The patterned electrode and separately prepared PDMS membrane are treated in oxygen plasma. E) The treated surfaces of the patterned electrode and PDMS membrane are placed in contact with each other to initiate bonding. F) The PVA substrate of the electrode is dissolved in hot water until the PVA is completely removed (up to 10 minutes), leaving the electrode permanently bonded to the PDMS.

\subsection{Fabrication Characterization}

With our laser system electrode line widths of less than $100 \mu \mathrm{m}$ are achievable for electrodes of thickness $\leq 8 \mu \mathrm{m}$ (Figure 1C). Significantly higher resolution (as low as $1 \mu \mathrm{m}$ ) should be achievable with laser technologies more optimized for high-resolution patterning, such as those using femto-second lasers. ${ }^{30,31}$

The bonding created as a result of the oxygen plasma treatment produce transducers with excellent electrode adhesion, as qualitatively demonstrated in Figure 3A using a "Scotch-tape" test. This results in mechanically robust devices as resistant to damage as the underlying elastomer (see Video S2 of the supporting information). Moreover the plasma exposure was found not to be detrimental to electrode resistance. One proposed mechanism for bonding is the reaction of oxygen radicals in the plasma with the methyl groups $\left(-\mathrm{CH}_{3}\right)$ of the PDMS chains, 
producing silanol $(-\mathrm{OH})$ groups in their place, followed by condensation reaction to form covalent bonds once the treated surfaces are in contact. ${ }^{32,34}$ After a period of approximately 30 minutes the PVA substrate is removed by dissolving in hot water. We are able to obtain good adhesion to a variety of commercially available PDMS membranes (listed in the supporting information) using our approach, providing great flexibility in transducer design.

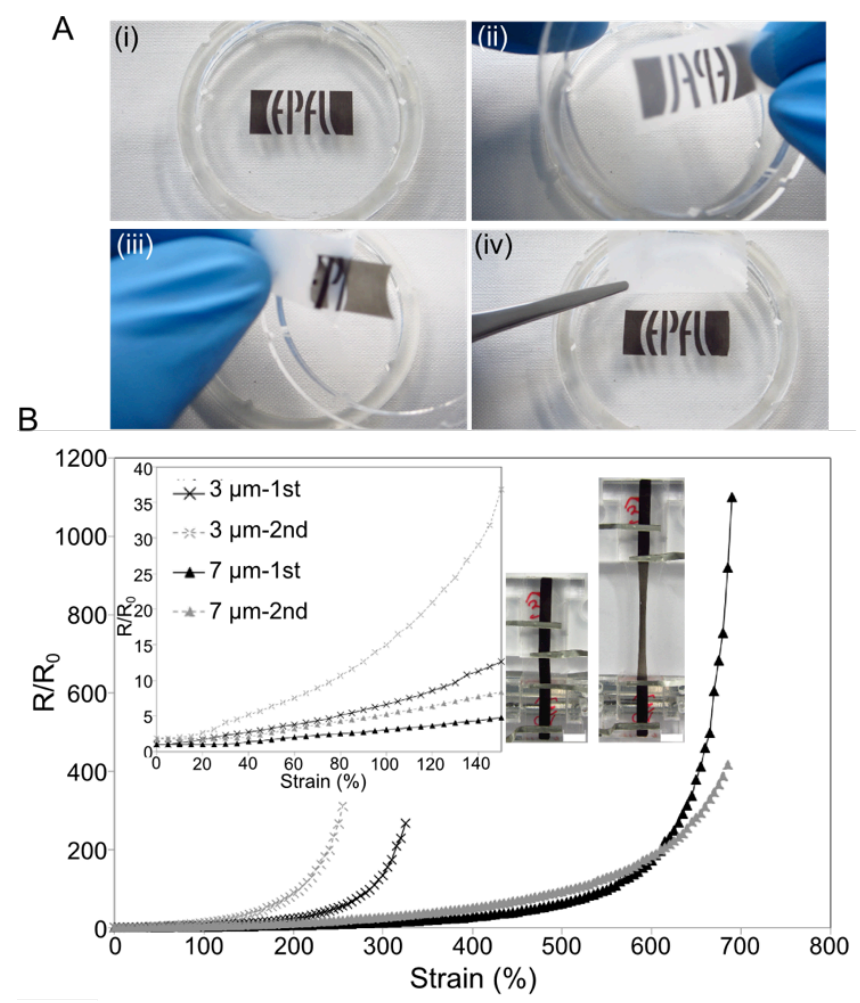

Figure 3. A) Demonstration of the high adhesion of a patterned electrode to elastomer membrane, using the "Scotch-tape" test. $3 \mu \mathrm{m}$ thick electrode patterned in the shape of the EPFL logo bonded to a $40 \mu \mathrm{m}$ thick PDMS membrane. (i) Electrode pattern before test (electrode bonded to back side of membrane), (ii) placing of adhesive tape on electrode surface, (iii) peeling off the tape, highly deforming the elastomer, (iv) electrode and elastomer are intact after tape removal. B) Electrode resistance versus strain for $3 \mu \mathrm{m}$ and $7 \mu \mathrm{m}$ thick electrodes for two strain cycles up to $700 \%$. Values normalized to the initial resistance of each electrode, 
approximately $300 \mathrm{k} \Omega$ and $100 \mathrm{k} \Omega$. Inset figures: zoom of strain region between $0-150 \%$ and images of the $7 \mu \mathrm{m}$ thick electrode sample at $40 \%$ and $600 \%$ strain (scale bar $=5 \mathrm{~mm}$ ).

Casting the electrode enabled the electrode thickness - and therefore conductivity and stiffening impact - to be easily tailored according to the application requirements with reasonable thickness homogeneity (typically thickness variation under $1 \mu \mathrm{m}$ ). Moreover, our methodology is compatible with various carbon powder fillers and filler ratios, providing additional design flexibility. Figure 3B shows the resistance variation against uniaxial strain of 3 $\mathrm{mm}$ wide and $5 \mathrm{~mm}$ long electrode samples cast to a thickness of $3 \mu \mathrm{m}$ and $7 \mu \mathrm{m}$ (initial resistances approximately $300 \mathrm{k} \Omega$ and $100 \mathrm{k} \Omega$ respectively). The results show that both electrodes remained conductive over strains of several hundred percent with the $7 \mu \mathrm{m}$ thick electrode remaining conductive at strains of over $600 \%$.

The maximum strain at which the electrodes lost conductivity was approximately double for the $7 \mu \mathrm{m}$ thick electrode compared to the $3 \mu \mathrm{m}$ electrode, implying a trade-off between stiffening impact of the electrode on the PDMS membrane (an important consideration when fabricating DEAs) and the maximum strain for which the electrodes remain conductive. Note that for both electrode thicknesses the electrodes remained conductive after the strain was removed and reapplied, though the resistance at zero strain had increased in both cases and the maximum strain for which the electrode remained conductive had decreased for the $3 \mu \mathrm{m}$ electrode. From a device perspective, this implies that a loss of conductivity resulting from extremely large externally applied deformations is non-fatal and that the conductivity will be recovered when the strain is released. 

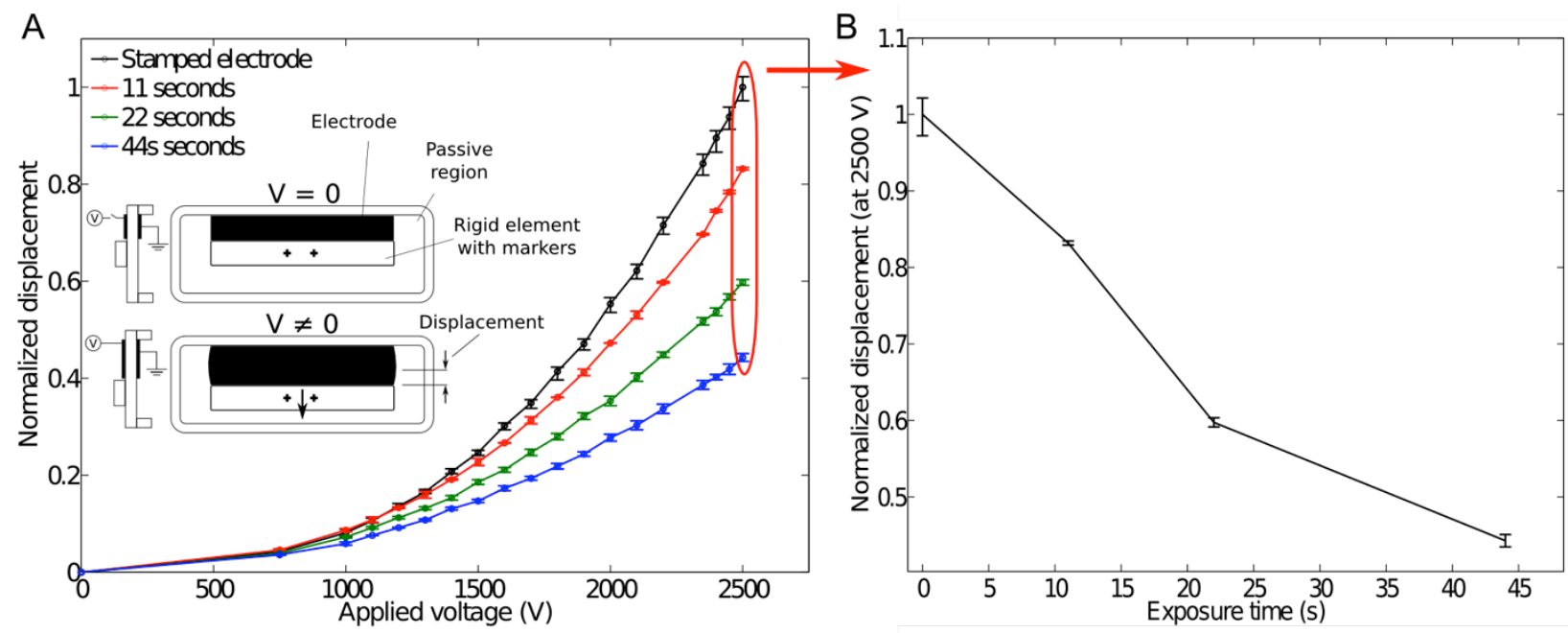

Figure 4. Actuation of DEAs showing the stiffening impact of oxygen plasma exposure. A) Actuator displacement for different plasma exposure times normalized by the displacement of the actuators with stamped (i.e. no plasma exposure) electrodes at $2.5 \mathrm{kV}$. Error bars are maximum and minimum values of the samples used to make the average. Inset shows actuator operating principle. B) Normalized actuator displacements from A) at an applied voltage of $2.5 \mathrm{kV}$ plotted as a function of plasma exposure time (data at zero seconds corresponds to stamped electrode). $11 \mathrm{~s}$ corresponds to the shortest time we used that allowed consistent high strength bonding, and retains $80 \%$ of maximum actuation strain compared to a plasma-free process.

Exposure to oxygen plasma is known to progressively produce a silica-like layer on the surface of the treated PDMS. ${ }^{35}$ This stiffens thin elastomer membranes and can thus reduce the strain produced by a DEA. We characterize this stiffening effect by measuring the actuation displacement generated by DEAs produced with different plasma exposure times using our technique. The actuator design is schematically shown in Figure 4A (inset), the device is 
optimized to produce in-plane, uni-directional displacement of a rigid element bonded to the prestretched membrane at its center, similar to the device reported in. ${ }^{36}$

The electrodes were bonded using plasma exposure times of 11, 22 and 44 seconds, and the results compared with similar electrodes stamped directly on the PDMS membrane (i.e. no plasma exposure). The results also show a monotonic decrease in the normalized actuation displacement produced by the actuators in the applied voltage range for increasing plasma exposure time. The normalized displacement at a drive voltage of $2.5 \mathrm{kV}$ vs. plasma exposure time, Figure 4B, shows an approximately linear reduction in displacement with exposure time. Plasma exposure times should be minimized, to minimize stiffening of the elastomer, but must be long enough to achieve sufficient bonding for a given application (11 s for our system).

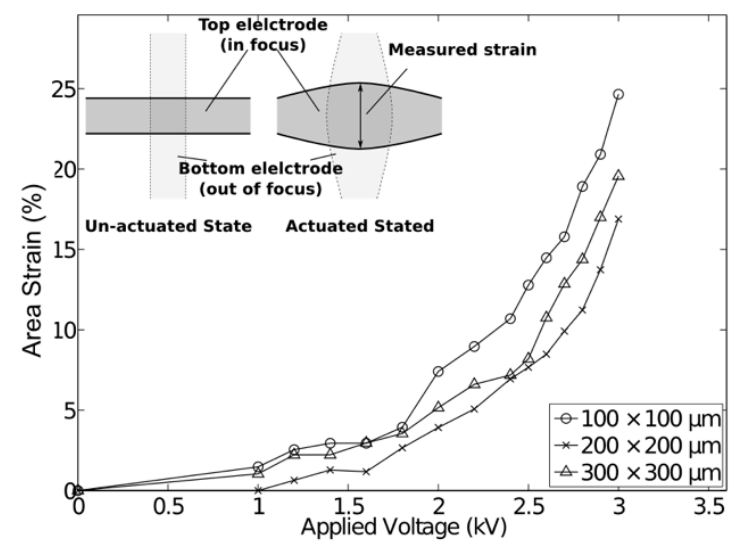

Figure 5. Cross-shaped DEA micro-actuators. Area strain vs. drive voltage for micro-actuators of dimension $300 \mu \mathrm{m} \times 300 \mu \mathrm{m}, 200 \mu \mathrm{m} \times 200 \mu \mathrm{m}$ and $100 \mu \mathrm{m} \times 100 \mu \mathrm{m}$ made using $3 \mu \mathrm{m}$ thick electrodes (plasma exposure time $=11 \mathrm{~s}$ for all devices).

3.2. Transducers with micro-scale features 
We use our approach to fabricate micro-sized DEAs, taking advantage of the high pattern resolution achievable by the laser ablation process. DEAs are seldom explored at the micro-scale though they have shown potential for the development of test assays for mechanotransduction studies of single or small biological cell populations ${ }^{37}$ and for tunable diffraction gratings. ${ }^{38}$ Micro-actuators of dimension $300 \times 300 \mu \mathrm{m}, 200 \times 200 \mu \mathrm{m}$ and $100 \times 100 \mu \mathrm{m}$ were fabricated by patterning orthogonal electrodes lines on opposite sides of a PDMS membrane (Figure 5). The actuators were made using the same PDMS and prestretch as those used for the plasma induced stiffening characterization in section 3.1. Area strains of up 25\% were achievable within the voltage range $0-3.0 \mathrm{kV}$, representing a safe operating range below the nominal breakdown field $=100 \mathrm{~V} / \mu \mathrm{m}\left(\right.$ from $\left.^{39}\right)$. These results show that functional micro-actuators can be produced using our technique, opening the door to further exploration of DEAs at the micro-scale. 

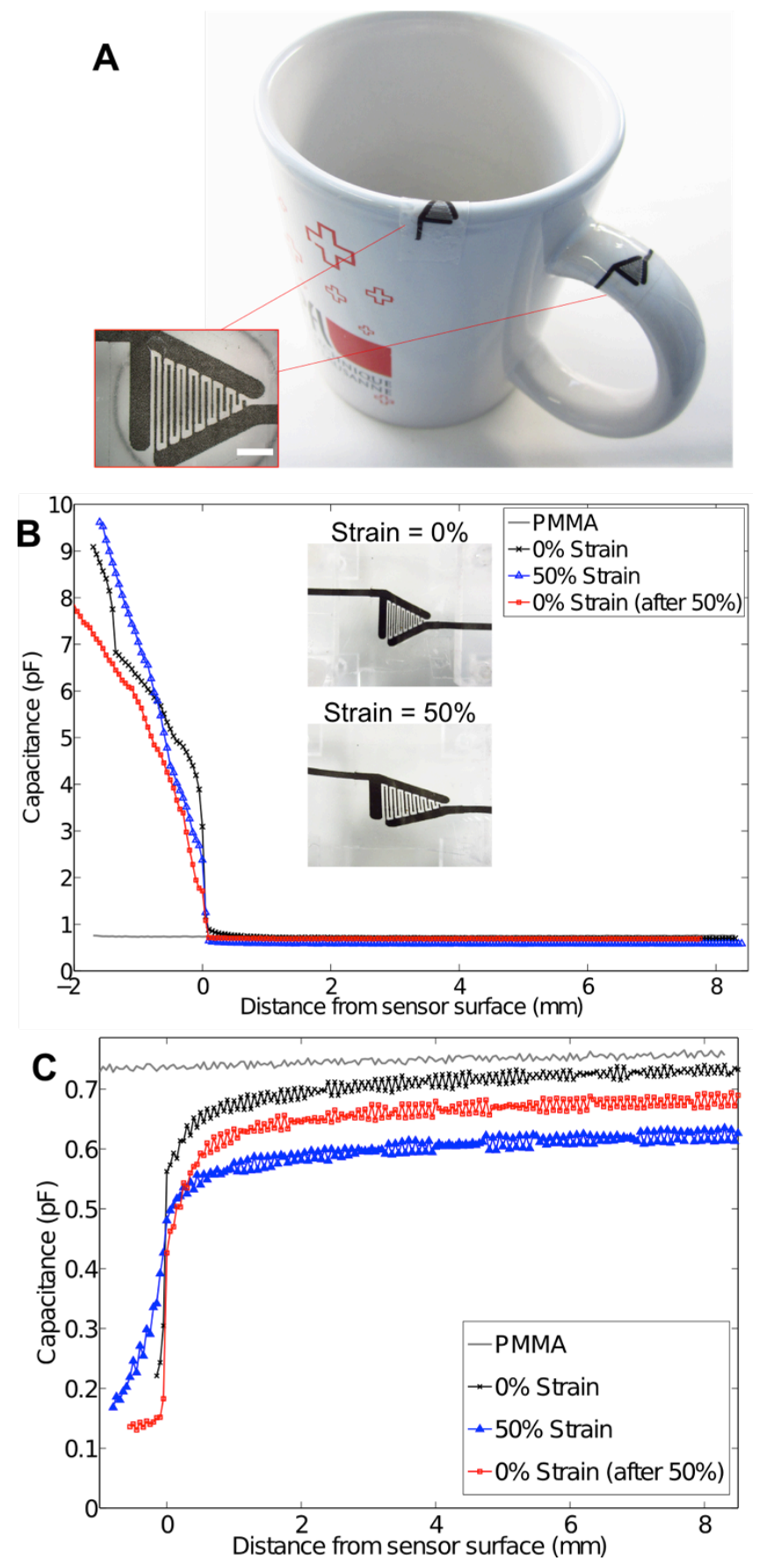

Figure 6. Interdigitated capacitive touch sensor with $300 \mu \mathrm{m}$ pitch. A) Demonstration of sensor conformability to both high curvature and low curvature regions of a coffee mug. Inset, close up image of sensor (scale bar $=2 \mathrm{~mm}$ ). B) Sensor capacitance vs. distance to a $13 \mathrm{~mm}$ diameter PDMS balloon filled with deionized water (high permittivity) approaching the sensor surface (inset, pictures of sensor at $0 \%$ and $50 \%$ strain). C) Sensor capacitance vs. distance to a $13 \mathrm{~mm}$ 
diameter PDMS balloon filled with a grounded salt-water solution. In B) and C) zero on the horizontal axis corresponds to the point of first contact of the balloon with the sensor. Sensor response to a PMMA probe (low permittivity) provided for comparison.

We fabricated an interdigitated capacitive sensor with $300 \mu \mathrm{m}$ pitch using our fabrication procedure (Figure 6A). The capacitance of the sensor changes in relation to the permittivity of the objects in its immediate vicinity, increasing when higher permittivity insulators are close, or decreasing for grounded conductors (i.e. the human body). ${ }^{3,11}$ Such sensors can be used for humidity sensing ${ }^{40}$ and can also be used to detect touch or finger proximity. The interdigitated design is advantageous from a fabrication point of view in that the sensing electrodes are coplanar, enabling device fabrication on one membrane surface with a single bonding step. Moreover, the co-planarity of the electrodes makes the device inherently vastly less sensitive to mechanical deformations of the elastomer membrane compared to typical parallel plate capacitive designs. The response of our sensor as a function of the distance of a PDMS balloon filled with deionized water (high permittivity insulator) and a grounded salt-water solution from the sensor surface is shown Figure 6 (experimental set up is shown in Figure S3 of the supporting information).

The results show an increase in the capacitance of at least a hundred percent between the point of initial contact (point at which separation distance $=0 \mathrm{~mm}$ ) and non-contact states for the deionized water experiment, and at least a $30 \%$ decrease for the grounded ionic solution at $0 \%$ strain. The sensor response increases further by several hundred percent compared to the baseline value as the deionized water balloon advances and covers more of the sensor area. The inverse 
effect can be seen with the grounded ionic solution balloon as it advances, with a capacitance reduction of almost $80 \%$ relative to the baseline value. These results indicate an increased sensitivity compared to stretchable parallel plate capacitive touch sensors of the same size scale $^{3,11}$ - the result of the interdigitated approach, further highlighting the utility of our highresolution patterning capability.

When the sensor is stretched by $50 \%$, the baseline capacitance value decreases by approximately $20 \%$, however the final values of capacitance are maintained with both the deionized water and grounded ionic solution. We draw the following conclusions from these results: 1) the sensor remains functional even when highly mechanically deformed, 2) the capacitance change relative to the baseline (unstretched) value is maintained even when stretched up to $50 \%$ uniaxially, and 3) even at these strains the change in baseline capacitance is lower than the change brought about by the stimuli, providing insensitivity to cross talk, which is an issue for stretchable parallel plate capacitive sensors. ${ }^{4}$ This therefore greatly increases the potential for achieving compact and high-resolution stretchable touch sensor arrays. Additionally, for applications requiring a high degree of conformability to an object (such as sensor skins) this property allows the sensors to be deformed and stretched around objects of various shapes and curvatures (Figure 6A) whilst remaining functional. Once the 50\% strain was released the baseline capacitance decreased slightly (probably due to viscoelastic creep) as seen in Figure 6C. However the sensor remained fully functional, with a sensitivity close to its initial value. 


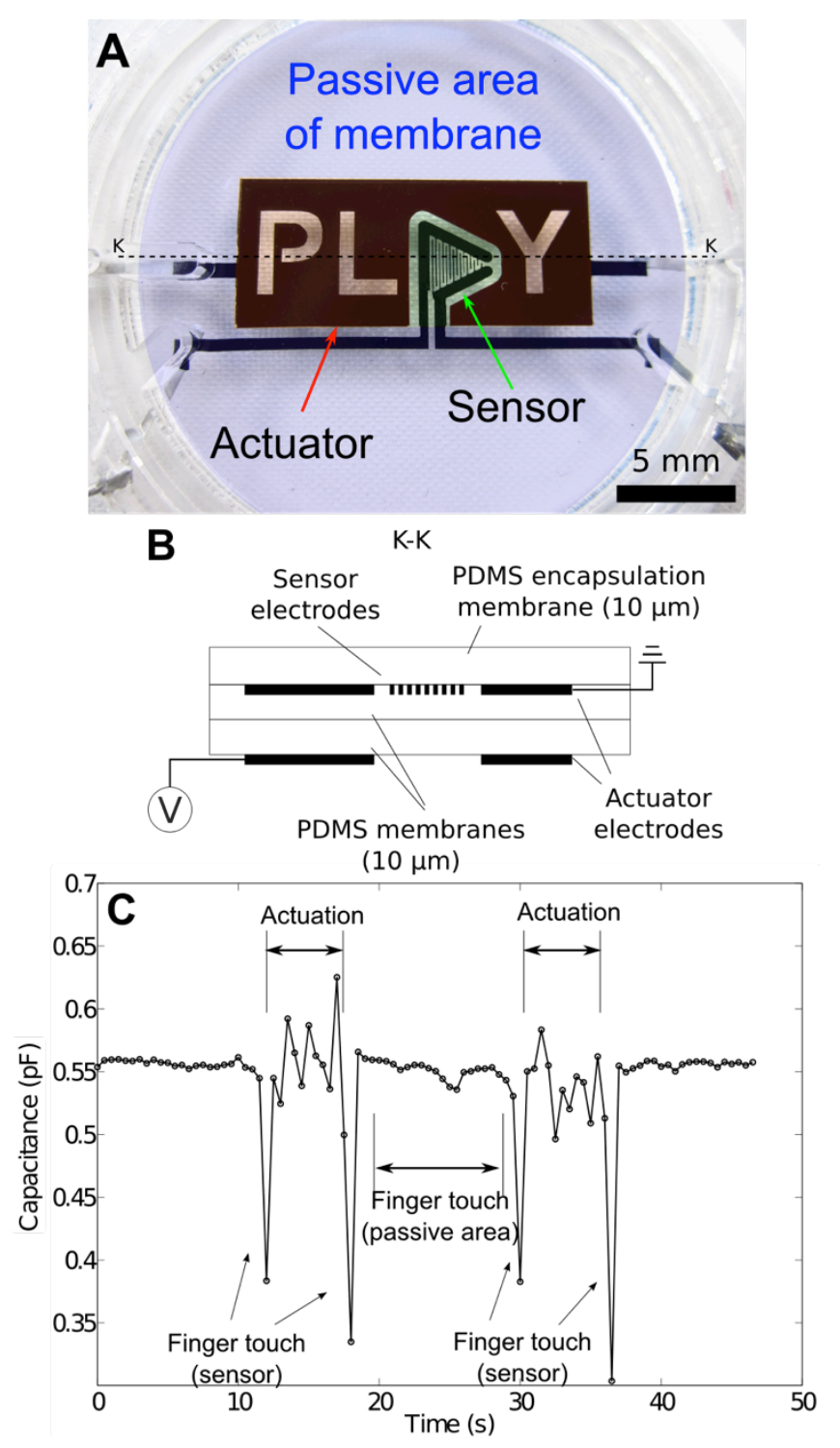

Figure 7. Stretchable capacitive proximity sensor and dielectric elastomer actuator made on the same elastomer membrane. An external circuit is used to read the capacitance and apply a square wave voltage to the DEA when a finger is detected, causing the play logo to pulsate until another finger touch is detected. A) Image of the device showing the actuator (shaded red) surrounding an interdigitated capacitive sensor (shaded green) and the passive area of the membrane (shaded blue). B) Device cross-section schematic. C) Response of sensor to finger touches directly on the sensor region. When the capacitance falls below $0.48 \mathrm{pF}$ (corresponding to a valid touch input) a $2 \mathrm{kV}$ periodic voltage input is applied to the actuator until a second touch input is detected. The 
sensor detects finger proximity, not strain: when the passive region of the membrane is touched, the device does not respond.

\subsection{Multifunctional transducers}

We further highlight the versatility of our fabrication method by producing a multifunctional demonstrator device capable of both touch sensing and actuation simultaneously (Figure 7). The touch sensor is an interdigitated capacitive sensor similar to that presented in section 3.2 , and the actuator (pulsing play logo) follows the DEA working principle explained in section 1 . The multifunctional device has a highly integrated design with the sensor located in the middle of the actuator region, resulting in a compact device (Figure 7A). The actuator ground electrode and capacitive sensor were patterned on the same electrode substrate and bonded to a prestretched elastomer membrane with a single plasma-bonding step.

Figure 7C shows the sensor response during device operation in which a user's touch input initiates actuation (see Video S3 of the supporting information). During actuation, small variations in the capacitance could be observed, related to the high electric fields used for actuation. However these variations were small compared to the touch input enabling subsequent touch inputs to be clearly discriminated. Touch inputs were also applied to the passive areas of the device membrane (Figure 7C), the device proved to be insensitive to these inputs, highlighting once again the sensor's impressive resilience to mechanical perturbations.

\section{Conclusion}


A novel fabrication methodology for high-resolution patterning of compliant electrodes for stretchable dielectric elastomer actuators and sensors was presented. The approach uses cast PDMS-carbon electrodes patterned by laser ablation and achieves robust permanent bonding to a silicone elastomer membrane by oxygen plasma activation. We demonstrate the flexibility of our methodology in fabricating a variety of transducer designs with sub-200 $\mu \mathrm{m}$ feature sizes over large areas (over $100 \mathrm{~cm}^{2}$ ). The process readily scales to much larger areas. The stiffening effect of the plasma exposure was characterized and shown to increase monotonically with exposure time. Excellent adhesion between the electrode and elastomer membranes can be achieved even with low (11 s) exposure times. Both functional micro-actuators and interdigitated capacitive sensors have been demonstrated with our technique. The capacitive sensor in particular showed excellent performance, maintaining high sensitivity even when stretched up to $50 \%$. Conversely the baseline change in capacitance was comparatively small even at these strains, providing insensitivity to mechanical perturbations and cross talk, and opening the potential to more compact stretchable touch sensor arrays. These results validate our technique as being a reliable and flexible platform from which to further explore micro-scale stretchable DEAs and DESs. We combined sensing and actuation in one multifunctional device demonstrator fabricated with the same process flow, combining high-resolution robust electrodes with large-area processing, showing the potential of our technique to produce sophisticated and highly integrated multifunction stretchable devices.

\section{ASSOCIATED CONTENT}


Supporting Information. Additional figures, experimental details and 3 videos demonstrating fabrication results. This material is available free of charge via the Internet at http://pubs.acs.org.

\section{AUTHOR INFORMATION}

\section{Corresponding Author}

*Email: seun.araromi@epfl.ch; herbert.shea@epfl.ch

\section{ACKNOWLEDGMENT}

We gratefully acknowledge the assistance of the members of the LMTS and the SAMLAB at EPFL, in particular Mr. Alexandre Poulin and Mr. Samuel Schlatter for their Labview scripts, and Ms. Nadine Besse, Dr. Andres Vásquez Quintero and Dr. Francisco Molina-Lopez for their help with sensor design. This work was partially funded by Swiss National Science Foundation grant \#200020_153122 and by the EPFL.

\section{REFERENCES}

(1) Bauer, S.; Bauer-Gogonea, S.; Graz, I.; Kaltenbrunner, M.; Keplinger, C.; Schwödiauer, R. 25th Anniversary Article: A Soft Future: From Robots and Sensor Skin to Energy Harvesters. Adv. Mater. 2014, 26 149-161. 
(2) Vandeparre, H.; Watson, D.; Lacour, S. P. Extremely Robust and Conformable Capacitive Pressure Sensors Based on Flexible Polyurethane Foams and Stretchable Metallization. Appl. Phys. Lett. 2013, 103, 204103.

(3) Cotton, D. P. J.; Graz, I. M.; Lacour, S. P. A Multifunctional Capacitive Sensor for Stretchable Electronic Skins. IEEE Sens. J. 2009, 9, 2008-2009.

(4) Yao, S.; Zhu, Y. Nanomaterial-Enabled Stretchable Conductors: Strategies, Materials and Devices. Adv. Mater. 2015, 27, 1480-1511.

(5) Pelrine, R.; Kornbluh, R.; Pei, Q. High-Speed Electrically Actuated Elastomers with Strain Greater than 100\%. Science. 2000, 287, 836-839.

(6) Akbari, S.; Rosset, S.; Shea, H. R. Improved Electromechanical Behavior in Castable Dielectric Elastomer Actuators. Appl. Phys. Lett. 2013, 102071906.

(7) Araromi, O. A.; Gavrilovich, I.; Shintake, J.; Rosset, S.; Richard, M.; Gass, V.; Shea, H. R. Rollable Multisegment Dielectric Elastomer Minimum Energy Structures for a Deployable Microsatellite Gripper. IEEE Trans. Mech. 2014, 20, 438-446.

(8) Shintake, J.; Rosset, S.; Schubert, B. E.; Floreano, D.; Shea, H. R. A Foldable Antagonistic Actuator. IEEE Trans. Mech. 2014, No. 99, 1-12.

(9) Rosset, S.; Shea, H. R. Flexible and Stretchable Electrodes for Dielectric Elastomer Actuators. Appl. Phys. A 2012, 281-307.

(10) Gisby, T. A.; O’Brien, B. M.; Anderson, I. A. Self Sensing Feedback for Dielectric Elastomer Actuators. Appl. Phys. Lett. 2013, 102, 193703. 
(11) Hu, W.; Niu, X.; Zhao, R.; Pei, Q. Elastomeric Transparent Capacitive Sensors Based on an Interpenetrating Composite of Silver Nanowires and Polyurethane. Appl. Phys. Lett. 2013, $102,083303$.

(12) Lotz, P.; Matysek, M.; Schlaak, H. F. Fabrication and Application of Miniaturized Dielectric Elastomer Stack Actuators. IEEE Trans. Mech. 2011, 16 58-66.

(13) Maffli, L.; Rosset, S.; Shea, H. R. Zipping Dielectric Elastomer Actuators: Characterization, Design and Modeling. Smart Mater. Struct. 2013, 22, 104013.

(14) Maffli, L.; Rosset, S.; Ghilardi, M.; Carpi, F.; Shea, H. Ultrafast All-Polymer Electrically Tunable Silicone Lenses. Adv. Funct. Mater. 2015, 25, 1656-1665.

(15) Michel, S.; Zhang, X. Q.; Wissler, M.; Löwe, C.; Kovacs, G. A Comparison between Silicone and Acrylic Elastomers as Dielectric Materials in Electroactive Polymer Actuators. Polym. Int. 2009, 59, 391-399.

(16) Benslimane, M.; Gravesen, P.; Group, M. T.; Danfoss, A. S.; Nordborg, D.-; Sommerlarsen, P. Mechanical Properties of Dielectric Elastomer Actuators with Smart Metallic Compliant Electrodes. In Electroactive Polymer Actuators and Devices (EAPAD) 2002, Proceedings of SPIE, San Diego, March 17-21, 2002; Yoseph Bar-Cohen, Ed.; SPIE: Bellingham, WA, 2011; 150.

(17) Niklaus, M.; Shea, H. R. Electrical Conductivity and Young's Modulus of Flexible Nanocomposites Made by Metal-Ion Implantation of Polydimethylsiloxane: The Relationship between Nanostructure and Macroscopic Properties. Acta Mater. 2011, 59, 830-840. 
(18) Rosset, S.; Niklaus, M.; Dubois, P.; Shea, H. R. Metal Ion Implantation for the Fabrication of Stretchable Electrodes on Elastomers. Adv. Funct. Mater. 2009, 19, 470-478.

(19) Corbelli, G.; Ghisleri, C.; Marelli, M.; Milani, P.; Ravagnan, L. Highly Deformable Nanostructured Elastomeric Electrodes with Improving Conductivity upon Cyclical Stretching. Adv. Mater. 2011, 23, 4504-4508.

(20) Yun, S.; Niu, X.; Yu, Z.; Hu, W.; Brochu, P.; Pei, Q. Compliant Silver NanowirePolymer Composite Electrodes for Bistable Large Strain Actuation. Adv. Mater. 2012, 24, $1321-1327$.

(21) Yuan, W.; Hu, L. B.; Yu, Z. B.; Lam, T.; Biggs, J.; Ha, S. M.; Xi, D. J.; Chen, B.; Senesky, M. K.; Grüner, G.; Pei, Q. Fault-Tolerant Dielectric Elastomer Actuators Using Single-Walled Carbon Nanotube Electrodes. Adv. Mater. 2008, 20, 621-625.

(22) Kubo, M.; Li, X.; Kim, C.; Hashimoto, M.; Wiley, B. J.; Ham, D.; Whitesides, G. M. Stretchable Microfluidic Radiofrequency Antennas. Adv. Mater. 2010, 22, 2749-2752.

(23) Tabatabai, A.; Fassler, A.; Usiak, C.; Majidi, C. Liquid-Phase Gallium-Indium Alloy Electronics with Microcontact Printing. Langmuir 2013, 29, 6194-6200.

(24) Park, M.; Park, J.; Jeong, U. Design of Conductive Composite Elastomers for Stretchable Electronics. Nano Today 2014, 9, 244-260.

(25) Keplinger, C.; Sun, J.-Y.; Foo, C. C.; Rothemund, P.; Whitesides, G. M.; Suo, Z. Stretchable, Transparent, Ionic Conductors. Science 2013, 341, 984-987. 
(26) Chen, B.; Lu, J. J.; Yang, C. H.; Yang, J. H.; Zhou, J.; Chen, Y. M.; Suo, Z. Highly Stretchable and Transparent Ionogels as Nonvolatile Conductors for Dielectric Elastomer Transducers. ACS Appl. Mater. Interfaces 2014, 6, 7840-7845.

(27) Michel, S.; Chu, B. T. T.; Grimm, S.; Nüesch, F. A.; Borgschulte, A.; Opris, D. M. SelfHealing Electrodes for Dielectric Elastomer Actuators. J. Mater. Chem. 2012, 22, 20736.

(28) Huang, J.; Lu, T.; Zhu, J.; Clarke, D. R.; Suo, Z. Large, Uni-Directional Actuation in Dielectric Elastomers Achieved by Fiber Stiffening. Appl. Phys. Lett. 2012, 100, 211901.

(29) Keplinger, C.; Li, T.; Baumgartner, R.; Suo, Z.; Bauer, S. Harnessing Snap-through Instability in Soft Dielectrics to Achieve Giant Voltage-Triggered Deformation. Soft Matter 2012, 8, 285-288.

(30) Wolfe, D. B.; Ashcom, J. B.; Hwang, J. C.; Schaffer, C. B.; Mazur, E.; Whitesides, G. M. Customization of Poly(dimethylsiloxane) Stamps by Micromachining Using a FemtosecondPulsed Laser. Adv. Mater. 2003, 15, 62-65.

(31) Buitinga, M.; Truckenmüller, R.; Engelse, M. A.; Moroni, L.; Ten Hoopen, H. W. M.; van Blitterswijk, C. A.; de Koning, E. J. P.; van Apeldoorn, A. A.; Karperien, M. Microwell Scaffolds for the Extrahepatic Transplantation of Islets of Langerhans. PLoS One 2013, 8, e64772.

(32) Bhattacharya, S.; Datta, a.; Berg, J. M.; Gangopadhyay, S. Studies on Surface Wettability of Poly(dimethyl) Siloxane (PDMS) and Glass under Oxygen-Plasma Treatment and Correlation with Bond Strength. J. Microelectromech. Syst. 2005, 14, 590-597. 
(33) Jo, B.-H.; Van Lerberghe, L. M.; Motsegood, K. M.; Beebe, D. J. Three-Dimensional Micro-Channel Fabrication in Polydimethylsiloxane (PDMS) Elastomer. J. Microelectromech. Syst. 2000, 9, 76-81.

(34) Mcdonald, J. C.; Duffy, D. C.; Anderson, J. R.; Chiu, D. T. Review - Fabrication of Microfluidic Systems in Poly (dimethylsiloxane). Electrophoresis 2000, 21, 27-40.

(35) Kim, J. Hydrophobic Recovery of Polydimethylsiloxane Elastomer Exposed to Partial Electrical Discharge. J. Colloid Interface Sci. 2000, 226, 231-236.

(36) Rosset, S.; Araromi, O. A.; Shea, H. R. Maximizing the Displacement of Compact Planar Dielectric Elastomer Actuators. Extrem. Mech. Lett. 2015, 3, 72-81.

(37) Akbari, S.; Shea, H. R. An Array of $100 \mu \mathrm{m} \times 100 \mu \mathrm{m}$ Dielectric Elastomer Actuators with 80\% Strain for Tissue Engineering Applications. Sens. Actuators A 2012, 186, 236-241.

(38) Aschwanden, M.; Stemmer, A. Low Voltage, Highly Tunable Diffraction Grating Based on Dielectric Elastomer Actuators. In Electroactive Polymer Actuators and Devices (EAPAD) 2007, Proceedings of SPIE, San Diego, March 19-22, 2007; Yoseph Bar-Cohen, Ed.; SPIE: Bellingham, WA, 2007; 65241N.

(39) Araromi, S.; Romano, P.; Rosset, S.; Perruisseau-Carrier, J.; Shea, H. A Tunable Millimeter-Wave Phase Shifter Driven by Dielectric Elastomer Actuators. In Electroactive Polymer Actuators and Devices (EAPAD) 2014, Proceedings of SPIE, San Diego, March 1013, 2014; Yoseph Bar-Cohen, Ed.; SPIE: Bellingham, WA, 2014; 90562M. 
(40) Molina-Lopez, F.; Briand, D.; De Rooij, N. F. All Additive Inkjet Printed Humidity

Sensors on Plastic Substrate. Sens. Actuators, B 2012, 166, 212-222. 


\section{Table of Contents Graphic}

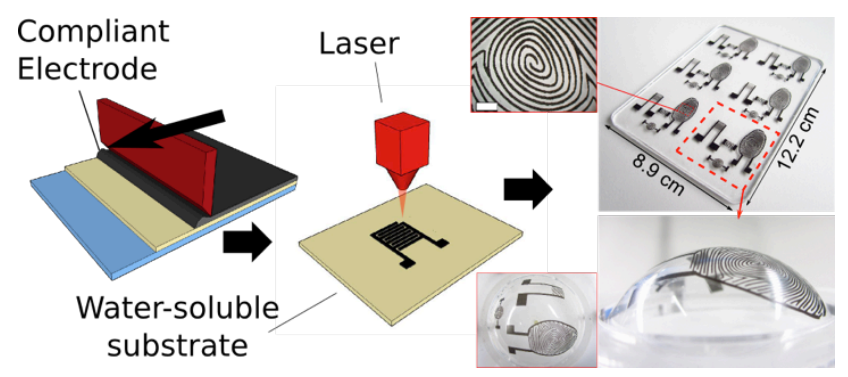

